\title{
Mobile energy monitoring system based on ZigBee communication
}

\author{
Qiao Yin Deng ${ }^{1, a^{*}}$ and Zhi Ping Wan ${ }^{2, b}$ \\ ${ }^{1,2}$ Xinhua College of Sun Yat-sen University , Guangzhou, China

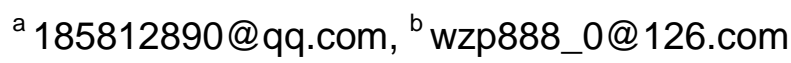

Keywords: ZigBee communication; Agricultural monitoring system; Mobile energy-efficient; Improvement of LEACH

\begin{abstract}
Objective: In order to achieve real-time to process and collect soil moisture, temperature and light data. Methods: To propose an Mobile energy monitoring system based on ZigBee communication. The system consists of a number of functional modules, each functional module the collected data sink base station, the base station sends data to each server, then the user management service through the remote access server. ZigBee sensor network system based in part on network design, communication module uses TI's new generation chip CC2530, ZigBee network protocol via RS232-RJ45 converter to access the LAN. Software algorithm to achieve improved LEACH data aggregation nodes and node communication. Results: The system is able to get the soil moisture and temperature and light data, and remote intelligent management. Conclusion: Through software emulation test verifies the ZigBee network communication stability and improved LEACH algorithm can be applied to the system.
\end{abstract}

\section{Introduction}

As China's aquaculture industry has been rapid development, has become the world's largest aquaculture country in an increasingly scarce natural resources and the environment, in order to achieve energy saving requirements and reduce labor costs, an urgent need for the establishment of an efficient conditions of industrial aquaculture systems. Effects of soil moisture due to crop root size, number and distribution[1], thereby affecting the growth and grain yield of the canopy, so the need for soil moisture monitoring, to ensure the normal growth of crops, but crop acreage is very broad, requires a lot of manual monitoring capital and labor costs[2].

ZigBee technology as one of the core technologies of wireless sensor networks in recent years before the rise of the short-range wireless communications technology, with low power consumption node device, a small communications interference, deployment speed, and a large network capacity and many other advantages, the technology can be well used in agricultural, environmental monitoring greenhouse crop growth control, reduce human factors affecting crop growth[3]. And ZigBee module by connecting a terminal module, users can remotely via Internet to receive / transmit signals to the ZigBee module, to obtain the required monitoring data or perform certain instructions. In order to achieve the same time, changes in farmland soil moisture-site and remote real-time monitoring, automatic watering and fertilizing, and make up for some reason suddenly fixed node failure situations[4]. This design as monitoring nodes in ZigBee wireless sensor networks, ZigBee module is also equipped with GPRS module, you can receive instructions from the terminal or in the case of failure of the other nodes via GPRS to the terminal transfer instructions can be accessed via the Internet soil moisture, nutrient and other data, to provide remote management support for agricultural irrigation and fertilization fine. 


\section{Mobile monitoring system development}

System Design. System hardware structure shown in figure. 1 Due to the distribution of a wide range of crops, remoteness, each monitoring point wired communication to lay cable or network lines, large installation costs need longer installation process. ZigBee node suitable for large-scale deployment, simple deployment, so our system monitoring points using ZigBee node deployment and load GPRS module, the node after the measurement data collected to measure the parameters passed to the terminal via a multi-hop manner, when unable to when passing through the multi-hop manner, then transferred via Ethernet. Power to the ZigBee wireless module which mobile nodes down to $3 \mathrm{~V}$ from $3.7 \mathrm{~V}$ lithium rechargeable battery by LDO chip SP6201-3.0. Systems underlying communication protocol for ZigBee protocol[5], nodes equipped with different types of sensors and actuators, networks, environmental information is uploaded to the ZigBee coordinator node. The coordinator node will receive data via the RS232-RJ45 protocol converter to convert the base station RJ45 upload format[6], while the wireless node via an IEEE 802.11 protocol video using different ports to send video data to the base station. Finally, the data shared by the base station to the site to monitor the server. Users then by calling the data server to view the test data of various types of sensors.

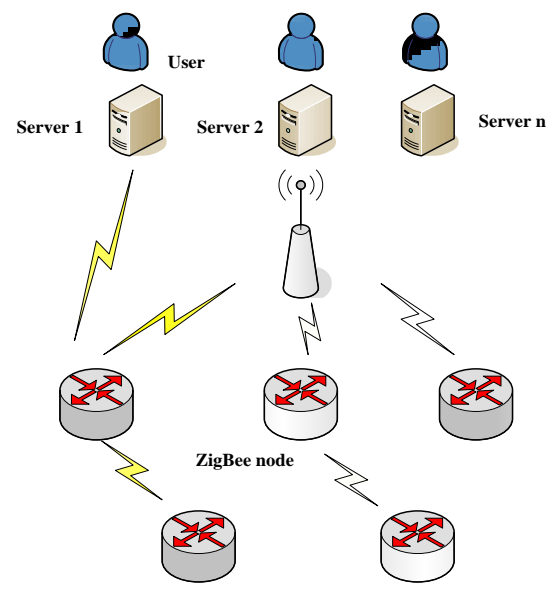

Figure. 1 System hardware structure

Hardware Design. CC2530 ZigBee communication module using the model system chip, this chip large capacity, suitable for ZigBeePro / 2007 complex protocol stack, CC2530 is a 2.4-GHz IEEE 802.15.4, ZigBee and RF4CE applications a true system-on-chip (SoC) solution. It can be a very low total cost of materials to build a strong network nodes. CC2530 combines the excellent performance of a leading RF transceiver, programmable flash memory industry-standard enhanced $8051 \mathrm{CPU}$, system, 8-KB RAM and many other powerful features[7]. There are four different flash CC2530 versions: CC2530F32 / 64/128/256, each having a 32/64/128 / 256KB flash. CC2530 has a different operating modes, making it especially adapted to the requirements of low power systems. The conversion time is running short between modes further ensure low energy consumption. CC2530 communication module structure diagram is shown below[8].

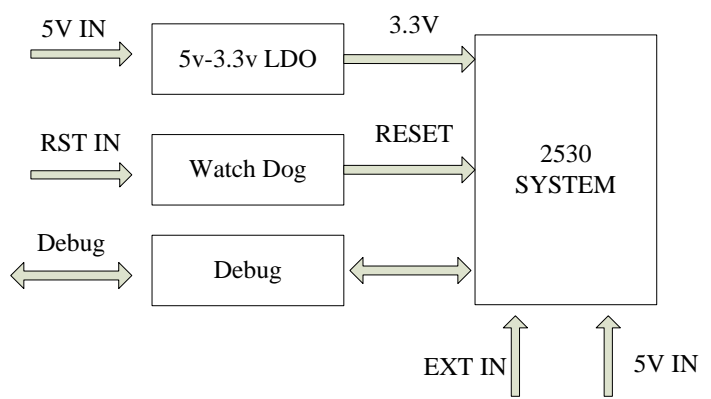

Figure. 2 CC2530 communication module structure 
Air temperature and humidity sensor we use high-precision Swiss Sensirion's two-wire serial digital temperature and humidity sensor SHT11, the temperature of the output resolution is 12 , the humidity value of the output resolution of 14 . Sensor supply voltage range of $2.4 \sim 5.5 \mathrm{~V}$. When measuring the current consumption is about $550 \mu \mathrm{A}$, the average $28 \mu \mathrm{A}[9]$, when dormant for $3 \mu \mathrm{A}$. Node can be set to timed acquisition, the acquisition is completed off the sensor power supply to further reduce power consumption. Light sensor HA2003 light sensors, the use of advanced photovoltaic module, the illumination intensity value is converted to a voltage value, and then the conditioning circuit converts this voltage $0 \sim 2 \mathrm{~V}$ or $4 \sim 20 \mathrm{~mA}$, high-precision measurement of light intensity compact, IP65 protection Level sensor design strong, corrosion-resistant, fast response, $<1$ second choice of voltage or current output, the current output when long cable transmission without signal attenuation[10].

Master chip STM32F405RG, Cortex-M4 processor has a high-performance 32-bit kernel, using the latest technology and a range of powerful low-power mixed-signal functions. It combines the efficient signal processing capabilities as well as the many incomparable advantages Cortex-M series processor and adding more innovative technology, up to 32 cores 1.25DMIPS / MHz; Thumb-2 instruction set to provide the best code density; and a nested vector interrupt controller, to complete the outstanding interrupt handling. STM32F405RG belong Cortex-M4 core chip that integrates a wealth of on-chip resources, up to 17-way timer, 12C, USART, SPI, a total of up to 15-way communication interface, but also to improve the data transfer rate of DMA channels supporting SWD and JTAG debug mode. SWD debug interface circuit from the power, ground, reset input, a clock and data line terminal end composed of a simplified model JTAG debugging, the actual debugging via JTAG debugger to debug.

Node power module uses TI's TPS63001DRC (3.3 V) and TPS63002DRC (5.0 V) 2 models offer power management chip voltage of 3.3 and $5.0 \mathrm{~V}$ systems. The system is designed AA5 No. 2 battery-powered or USB power supply, reserve lithium battery interface. Seamlessly switch between power supply.

Software Design. Coordinator software flow chart shown in figure 3. The coordinator position after power up sends equipment identification message after receiving the response into the loop to wait until the PC operating instructions issued to a node, the coordinator after receiving the command to enter the message processing loop, disposed of receiving PC application after disconnecting command disconnected. The ZigBee coordinator after power, do the following: 1) initialization of the network, including the initialization ZigBee protocol stack initialization and peripherals; 2) channel query, select the appropriate channel, waiting for node joins the network; 3) with the main function module for serial communications, in order to achieve real-time display and store the data terminal, while receiving node receives commands from the serial port on the terminal user input and send to the corresponding node the command. PC monitoring software is based on a modular design, aside interface. This system is designed to facilitate the further escalation of Debug and post software. Software design is based on large-scale systems theory thinking on various factors throughout the greenhouse group environment into consideration, the social management system, taking into account factors such as design, according to the experts to provide basic crops crop growth parameters will simply preset crop growth model into client. 

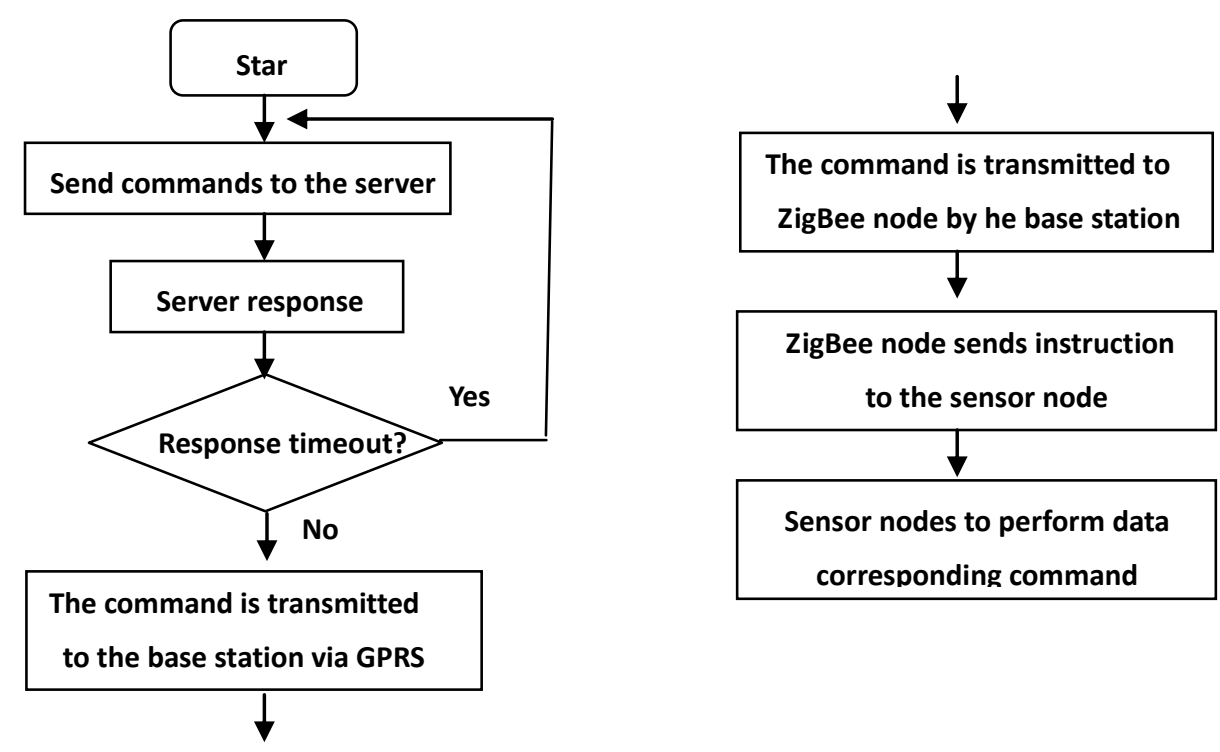

Figure. 3 command transmission flow chart

\section{System testing and analysis}

Test scenarios in this paper for three areas of agricultural land approximately $2000 \mathrm{~m} 2$ of uniformly distributed 20 ZigBee nodes. In each round selection from the work ZigBee cluster head, base station node needs to do more communication and data processing, more energy, so a power supply, set up in the nearby crops. The 1st Field using conventional LEACH protocol, each node is selected as the cluster head rotation, setting $20 \mathrm{~s}$ per node sends data to the cluster head; the 2nd field improved LEACH protocol, node battery energy monitoring, so that the battery power supply much more likely to be selected as the node cluster head; By monitoring the number of nodes failure shown in figure 4 , it is assumed to reach 10 when the failed node Zigbee network paralysis, improved LEACH protocol networks than conventional LEACH protocol extending the $30 \%$, the threshold is set to optimize the effective time, reaching the node balanced and objective to conserve battery power remaining battery energy.

Analysis of the data we can see that at close range low packet loss rate, and when the communication distance becomes greater than $400 \mathrm{~m}$, packet loss rate in excess of $10 \%$, as shown in figure 5. This is due to the low-power communication link module has great irregularities, node communication link quality is divided into an active area, transition area and the blank area may be within $200 \mathrm{~m}$ signal transition region, data loss caused by the communication signal is unstable increased rate. And about $400 \mathrm{~m}$ off due to environmental factors scattering wireless signal, a signal superposition, but enhanced communication effectiveness, and then gradually into the blank area of signal loss rate increases. . LEACH protocol which uses improved compared to LEACH protocol can reduce packet loss rate.

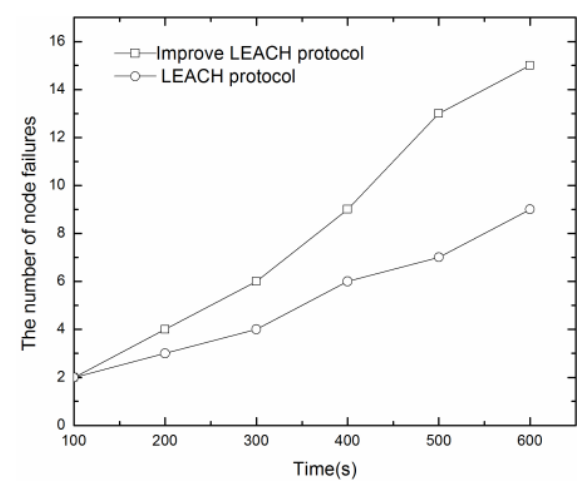

Fig. 4 Schematic of failed node

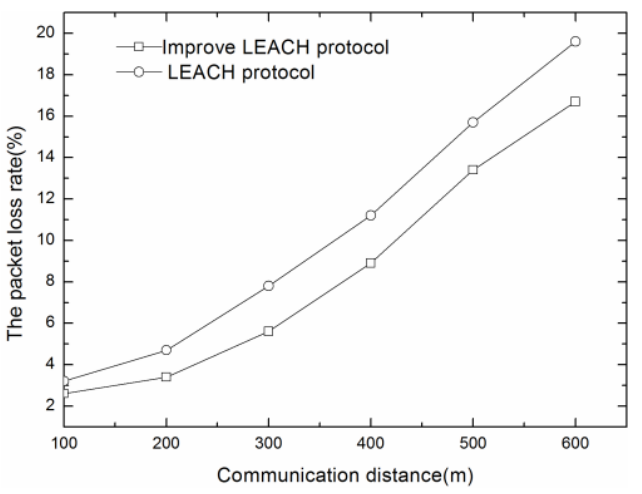

Fig. 5 The packet loss rate 


\section{Summary}

Designed to move energy-saving agricultural monitoring system based on ZigBee communications, energy and other growing areas of farmland on air temperature, humidity, light, real-time acquisition, real-time control of sensors to good effect.

Node communication tests showed that under normal operating conditions, an improved LEACH algorithm, 200m communication packet loss rate was $3.4 \%$, 400m less than $8.9 \%$, to fully meet the needs of engineering design, ad hoc network nodes can, and to achieve a certain degree of energy efficiency effect.

The sensor peripheral circuit and power supply modules need to be further optimized, reducing the node size, reduce node power consumption.

\section{References}

[1] Han D M, Lim J H. Smart home energy management system using IEEE 802.15. 4 and zigbee[J]. Consumer Electronics, IEEE Transactions on, 2010, 56(3): 1403-1410.

[2] Gao L, Lan Y D. Energy Saving Application of Data Fusion Algorithm in ZigBee Networks[J]. International Journal of Applied Mathematics and Statistics ${ }^{\mathrm{TM}}$, 2014, 52(2): 86-97.

[3] Razouk W, Crosby G V, Sekkaki A. New Security Approach for ZigBee Weaknesses[J]. Procedia Computer Science, 2014, 37: 376-381.

[4] Samuel C P J, Prakash S, Kumar M A, et al. ZigBee Based Home Security Alert System: An Integrated Approach[J]. Journal of Artificial Intelligence, 2013, 6: 89-94.

[5] Amir S, Choi B, Choi Y J. Interference coordination between Wi-Fi and ZigBee networks[C]//ICT Convergence (ICTC), 2013 International Conference on. IEEE, 2013: 728-729.

[6] Reddy C H, Reddy G K. Image Processing based Energy Meter Reading using ZigBee and GSM[J]. International Journal of Applied Engineering Research, 2014, 9(18): 4997-5005.

[7] Liang G, Zhou N, Xu W, et al. ZigBee Based Slope Wireless Monitoring Networks[J]. DISASTER ADVANCES, 2013, 6: 151-157.

[8] Baig F, Beg S, Khan M F. Zigbee based home appliances controlling through spoken commands using handheld devices[J]. International Journal of Smart Home, 2013, 7(1): 19-26.

[9] Shen J M, Wei H L. Circuit Design of Panel Points Based on Zigbee[M]//Informatics and Management Science III. Springer London, 2013: 3-9.

[10] Peng S, Cui B, Jia R, et al. A novel vulnerability detection method for ZigBee MAC layer[J]. International Journal of Grid and Utility Computing, 2013, 4(2): 134-143. 